\title{
Adaptive Shadow and Highlight Invariant Colour Segmentation for Traffic Sign Recognition Based on Kohonen SOM
}

\author{
Hasan Fleyeh and Al-Hasanat R. M. Bin Mumtaz
}

\begin{abstract}
This paper describes an intelligent algorithm for traffic sign recognition which converges quickly, is accurate in its segmentation and adaptive in its behaviour. The proposed approach can segment images of traffic signs in different lighting and environmental conditions and in different countries. It is based on using Kohonen's Self-Organizing Maps (SOM) as a clustering tool and it is developed for Intelligent Vehicle applications. The current approach does not need any prior training. Instead, a slight portion, which is about $1 \%$ of the image under investigation, is used for training. This is a key issue to ensure fast convergence and high adaptability. The current approach was tested by using 442 images which were collected under different environmental conditions and from different countries. The proposed approach shows promising results; good improvement of $73 \%$ is observed in faded traffic sign images compared with $53.3 \%$ using the traditional algorithm. The adaptability of the system is evident from the segmentation of the traffic sign images from various countries where the result is $96 \%$ for the nine countries included in the test.
\end{abstract}

Keywords. Colour segmentation, neural networks, traffic signs, recognition, classification, SOM.

2010 Mathematics Subject Classification. 68T10, 68T45.

\section{Introduction}

Colour represents an important part of the information provided to the driver to ensure the objectives of the traffic signs. Therefore, traffic signs and their colours are selected to be different from the natural environment or from the surrounding in order to be distinguishable. Detection of these signs in outdoor images from a moving vehicle will help the driver to make the right decision in good time, which means fewer accidents, less pollution, and better safety.

One of the important steps in Traffic Sign Recognition (TSR) is Segmentation. It is the process by which candidate objects are specified for further analysis according to certain properties such as colour or shape. Colour segmentation has always been considered a strong tool for image segmentation because it is com- 
putationally inexpensive. Due to the fact that colour gives more information than grey it is used in segmentation algorithms instead of edge-based and luminance histogram-based techniques.

There are many segmentation algorithms available for traffic sign recognition in the literature. However, none of them has tackled the problem of adaptability, which is a need because segmentation of traffic signs in outdoor images may face one or more of the following problems. Firstly, the apparent colour of the object varies because of the chromatic variation of daylight. Secondly, colours of traffic sign boards change with age which means newly installed traffic signs have different colours than older traffic signs. Thirdly, different countries use different standard colours for traffic signs. In addition to this, traffic sign images may suffer from the effect of shadows and highlights. The effect of shadows occurs when different parts of the object are exposed to different illumination levels, while in the case of highlight the object reflects some of the light of the illuminant directly to the viewer.

The aim of this paper is to present an adaptive colour segmentation algorithm which is immune to the effect of shadows and highlight, it can work under the circumstances mentioned above, and it shows fast convergence. The proposed approach invokes Kohonon's self-organizing maps (SOM) [7] as a tool. It is an unsupervised learning method which clusters data (in this case colour) on the basis of the given features to the network. The information can thus be received from the Best Matching Units (BMU's) obtained from SOM.

The reminder of the paper is organised as follows. In the next section, the relevant work is introduced. Challenges are presented in Section 3. In Section 4 the system design is illustrated. The experiments and results based on the proposed method are given in Section 4, and in Section 5 the conclusions are presented.

\section{Relevant Work}

In recent years, research into traffic sign recognition has grown rapidly because of the real need for such systems not only for future vehicles but also for the current in-vehicle assistant systems. Performance indexes necessary for these systems include high recognition rates, real-time implementation, many traffic sign categories as recognition objects, robustness for variant environments, and feasibility under poor visibility conditions.

Fleyeh [4] presented a colour detection and segmentation algorithm for traffic signs in which the effect of shadows and highlights is targeted using HSV colour space. The segmentation algorithm was invariant to shadows and highlights. Soetedjo and Yamada [9] proposed a traffic sign recognition system using a thresh- 
olding method based on CIE-RGB chromaticity diagram. Prieto and Allen [8] developed a method to detect potential road signs by analysing the distribution of red pixels using SOM. According to this method, the potential road signs were detected by analysing the distribution of red pixels in an image, and then road signs were identified from the distribution of dark pixels in their pictograms.

In addition to traffic sign recognition, SOM has been used as a tool for colour segmentation in general. Yeo et al. [12] proposed a competitive-learning neural network model for colour image segmentation based on SOM and the Adaptive Resonance Theory (ART). The LARSOM developed by Araujo and Costa [1] provides strong footing for the segmentation with SOM while claiming good results for colour image segmentation. Wu et al. [11] presented an adaptive selforganising colour segmentation algorithm which is used to localise human hands in video sequences.

\section{Challenges}

\subsection{Colour Variations in Outdoor Images}

One of the most difficult problems in using colours in outdoor images is the chromatic variation of daylight which causes the apparent colour of the object to vary as daylight changes. The irradiance of any object in a colour image depends on three parameters:

The colour of the incident light: Daylight's colour varies along the CIE curve. It is given by:

$$
y=2.87 x-3.0 x^{2}-0.275 \text { for } 0.25 \leq x \leq 0.38 .
$$

The variation of daylight's colour is a single variable which is independent of the intensity.

The reflectance properties of the object: The reflectance of an object $s(\lambda)$ is a function of the wavelength $\lambda$ of the incident light. It is given by:

$$
s(\lambda)=e(\lambda) \phi(\lambda)
$$

where $e(\lambda)$ is the intensity of the light at wavelength $\lambda$, and $\phi(\lambda)$ is the object's albedo at each wavelength. 
The camera properties: The observed intensities depend on the lens diameter $d$, its focal length $f$, and the image position of the object measured as angle $a$ off the optical axis. This is given by:

$$
E(\lambda)=L(\lambda) \cdot(\pi / 4)(d / f)^{2} \cos (4 a) .
$$

According to this equation, the radiance $L(\lambda)$ is multiplied by a constant which will not affect an object's observed colour. By cancelling the camera's lens chromatic aberration, only the density of the observed light will be affected.

As a result, the colour of the light reflected by an object located outdoors is a function of the temperature of the daylight and the object's albedo [2], [3].

\subsection{Aging of Traffic Signs}

Traffic signs may be mounted on a pole for a long time without any kind of maintenance or replacement. Over time the properties of the material used to reflect the light in these traffic signs changes its properties because of environmental reactions and so its colour fades. Figure 1 shows two traffic signs; the sign on the left is a new one while the one on the right is an old one.

To investigate the effect of aging, 30 traffic signs were selected. Half of this set consisted of new traffic signs and the other half contained old ones. Colour located in the red part of each traffic sign was extracted and converted from RGB into HSV. HSV was selected because it is invariant to the variations in light conditions as it is multiplicative/scale invariant, additive/shift invariant, and it is invariant under saturation changes. In addition it has been proven by Gevers and Smeulders [6] that hue is invariant against shadow and highlights.

Vitabile et al. [10] defined three different areas in the HSV colour space:
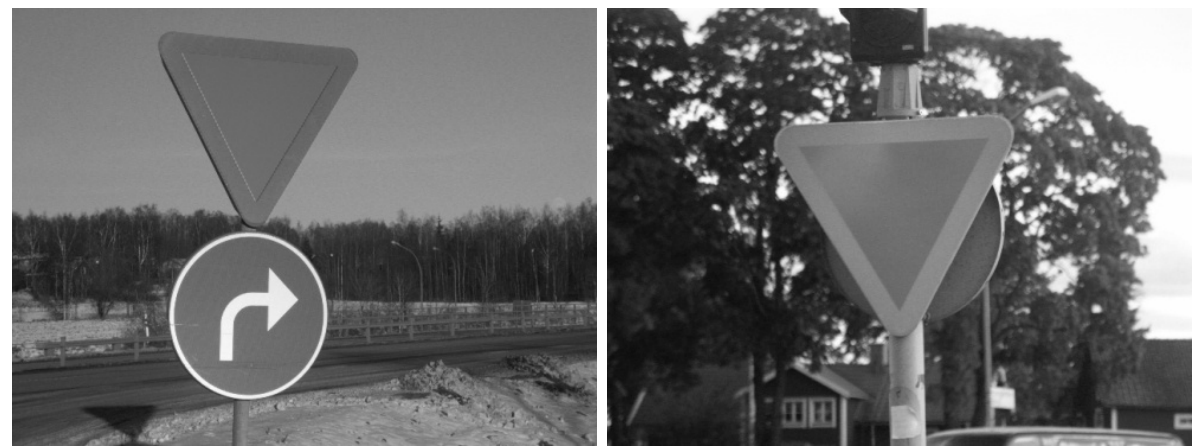

Figure 1. Effect of aging. Left: a new traffic sign. Right: an old traffic sign. 


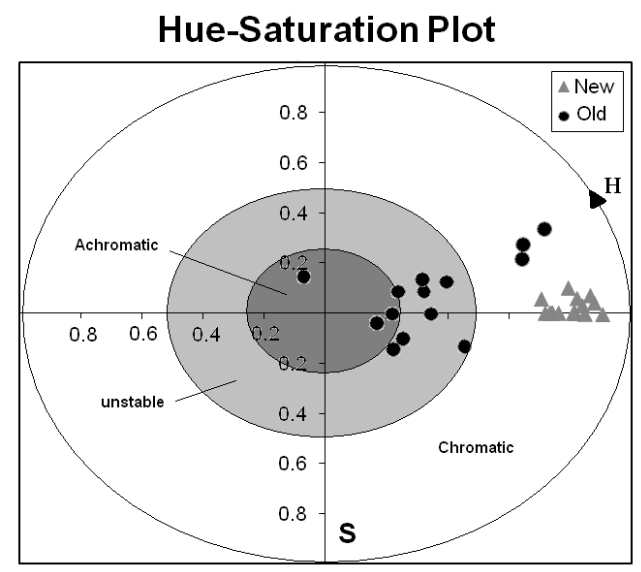

Figure 2. Hue-saturation plot of new and old traffic signs.

- The achromatic area: characterised by $s \leq 0.25$ or $v \leq 0.2$ or $v \geq 0.9$.

- The unstable chromatic area: characterised by $0.25 \leq s \leq 0.5$ and $0.2 \leq$ $v \leq 0.9$.

- The chromatic area: characterised by $s \geq 0.5$ and $0.2 \leq v \leq 0.9$.

Plotting the hue and saturation of each traffic sign in the hue-saturation plot is shown in Figure 2. The new traffic signs were grouped in the chromatic region while old traffic signs shifted either towards the yellow part of the hue or towards the unstable or even towards the achromatic area of this plot. Old traffic signs which moved toward the yellow but still in the chromatic area can be colour segmented, while the colour of the remaining traffic signs cannot be segmented because of the loss of hue.

\subsection{Traffic Signs from Different Countries}

Different countries use different colours for their traffic signs. Figure 3 shows two yield traffic signs from The Netherlands (left) and from Sweden (right). To understand the distribution of traffic signs from different countries on the huesaturation plot, a number of traffic signs were collected from some countries in Europe, from the USA and Japan. Plotting the red colour on the hue-saturation plot clearly indicates that the red colour of these traffic signs is grouped in a certain region in this plot. 

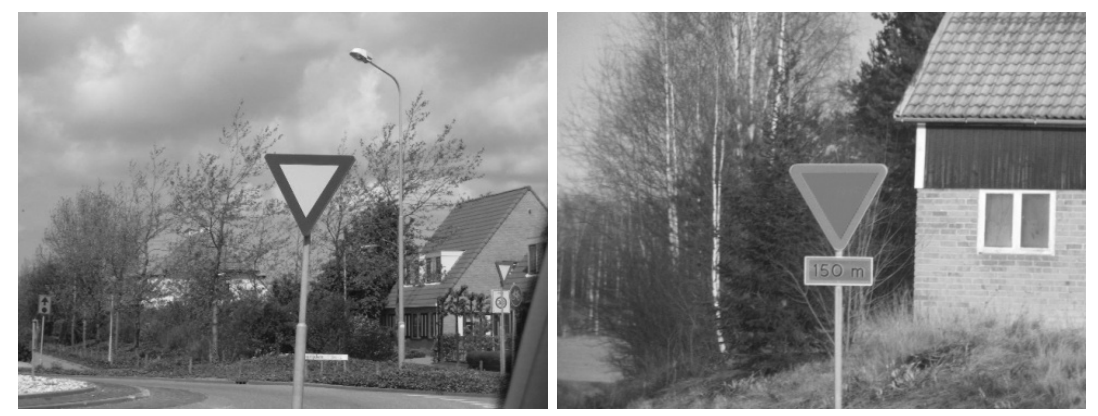

Figure 3. Colours used by different countries: left, The Netherlands; right, Sweden.

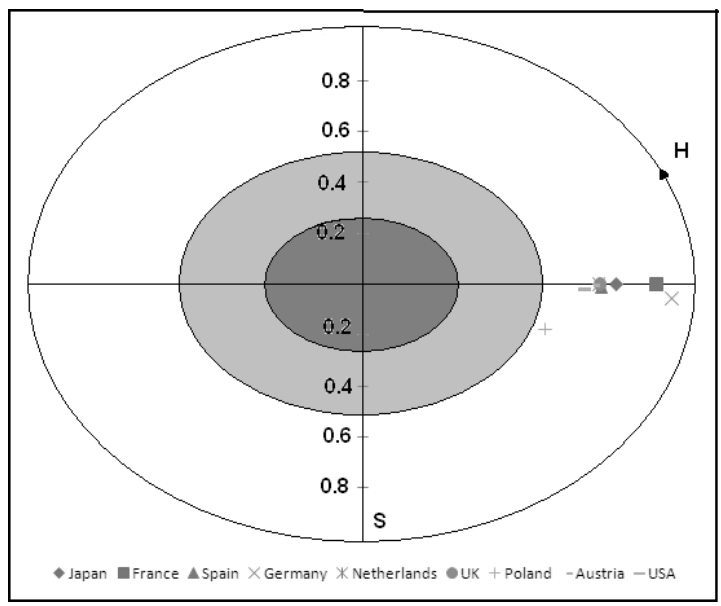

Figure 4. Hue-saturation of traffic sign colours of different countries. Colours used by different countries: left, The Netherlands; right, Sweden.

\section{System Design}

SOM [7] is a powerful unsupervised clustering technique which can be utilised to solve the problem of colour segmentation. Colour segmentation is based on reducing the number of colours in an image. It is achieved by selecting the most representative colours from all colours present in the original image, making a colour palette and then mapping each colour in the image to the nearest colour in the palette. Since each colour in the segmented image represents an object, only a few colours are desired in the colour palette. 


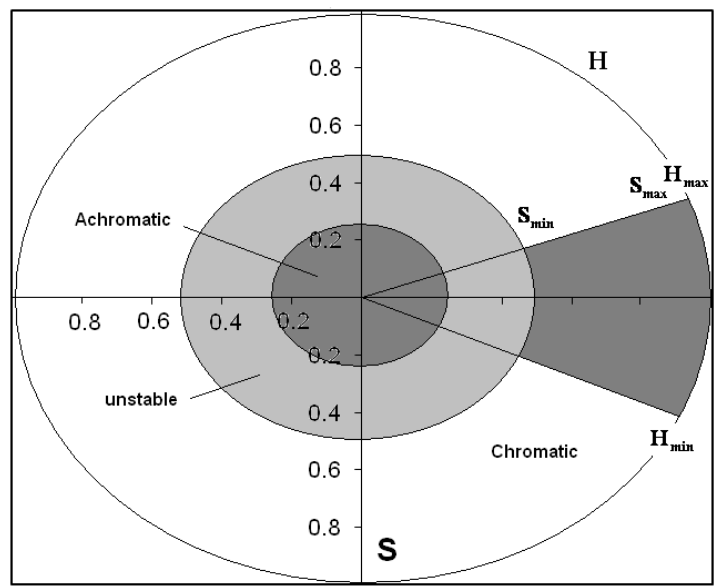

Figure 5. Dynamic Search Boundary by the proposed algorithm for the red colour.

An important feature on which the quality of segmentation depends is the search space boundaries. In-depth analysis was carried out on traffic sign images in different conditions for colours chosen for segmentation. For the reasons mentioned in Section 3.2, HSV colour space was utilised as a basic colour cue. RGB images were converted into HSV colour space and the $H$ and $S$ values of the traffic signs were observed to assign a reasonable search boundary in the $H$ and $S$ space. Since the values of $H$ and $S$ differ from one traffic sign to another (for the reasons mentioned in Section 3), it is not wise to choose static boundaries. Instead, boundaries of a search subspace in the $H-S$ plot are specified dynamically by the SOM which is trained to find the upper and lower limits of $H$ and $S$ through the BMU's. It is, therefore, believed that the algorithm presented in this paper is adaptive and can be used in a wide range of environmental conditions and in different countries.

The values $H_{\min }, H_{\max }, S_{\min }$, and $S_{\max }$ in Figure 5 are specified dynamically by the SOM. This means that the position and the width of the search space dynamically depend on the desired colour of segmentation.

The adaptive segmentation algorithm for traffic sign recognition is given in the following steps.

Step 1: Read the image to be segmented in RGB and convert it into HSV.

Step 2: Parameters initialisation

Step 2.1: Specify the initial values of $H_{\min }, H_{\max }, S_{\min }$, and $S_{\max }$ of the colour to be segmented. 
Step 2.2: Initialise the size of the grid $\mathrm{G}$ such that the number of nodes in the network $\mathrm{N}$ is given by $N=G^{2}$.

Step 2.3: Initialise the time iteration, $t=0$; the neighbourhood variance, $\sigma_{0}$, the learning rate, $\eta_{0}$, the maximum number of epochs for training, and the minimum allowable error $e_{\min }$. Typical values of $\sigma_{0}$ is $G / 2, \eta_{0}$ is 0.2 and $\mathrm{e}_{\min }$ is $10^{-3}$.

Step 3: Create two weight matrices $\mathbf{W}_{\mathbf{H}} \in \mathbb{R}^{G \times G}$ as the hue weight matrix and $\mathbf{W}_{\mathbf{S}} \in \mathbb{R}^{G \times G}$ as the saturation weight matrix between input vector and the nodes in the grid.

$$
\mathbf{W}_{\mathbf{H}}=\left[\begin{array}{cccc}
w_{h(1,1)} & w_{h(2,1)} & \cdots & w_{h(G, 1)} \\
w_{h(1,2)} & w_{h(2,2)} & \cdots & w_{h(G, 2)} \\
\vdots & \vdots & \ddots & \vdots \\
w_{h(1, G)} & w_{h(2, G)} & \cdots & w_{h(G, G)}
\end{array}\right], \quad \mathbf{W}_{\mathbf{S}}=\left[\begin{array}{cccc}
w_{s(1,1)} & w_{s(2,1)} & \cdots & w_{s(G, 1)} \\
w_{s(1,2)} & w_{s(2,2)} & \cdots & w_{s(G, 2)} \\
\vdots & \vdots & \ddots & \vdots \\
w_{s(1, G)} & w_{s(2, G)} & \cdots & w_{s(G, G)}
\end{array}\right]
$$

The weight vector for any node located at $i, j$ in the grid is given by

$$
\mathbf{w}_{i, j}=\left[w_{h(i, j)} w_{s(i, j)}\right]^{T} .
$$

Step 4: Initialise the two matrices in step 3 randomly such that every element in the matrix is in the interval $[0,255]$.

Step 5: Randomly choose pixels from the image to be segmented and perform the following sub steps:

Step 5.1: Present the input vector $\mathbf{x}=\left[\begin{array}{ll}H S & S\end{array}\right]^{T}$ of the image to be segmented to the SOM. The input vector is the $H$ and $S$ values of the randomly chosen pixel.

Step 5.2: Find the Euclidean distance $D_{i, j}$ between the input vector and the corresponding node located at $(i, j)$ in the grid using the following equation:

$$
D_{i, j}^{2}=\left\|H-w_{h(i, j)}\right\|^{2}+\left\|S-w_{s(i, j)}\right\|^{2} .
$$

Step 5.3: On the basis of distance, select the node which is closest to the input pattern, the smaller the distance the closer that node is from the input pattern.

Step 5.4: Update the weights of the winning node and its neighbourhood nodes on the basis of the distance from the winning node and exclude all other nodes from weight updating. 
Let $d_{j, i}$ denote the lateral distance between the winning neuron $i$ and the excited neuron $j$ both of which are measured in the discrete output space and $h(t)$ denote the neighbourhood function given by

$$
h(t)=\exp \left(\frac{d_{j, i}}{2 \sigma^{2}(t)}\right) .
$$

The weight vector of the BMU and its neighbours are updated as follows:

$$
\begin{aligned}
& w_{h(i, j)}(t+1)=w_{h(i, j)}(t)+\eta(t) h(t)\left(H-w_{h(i, j)}(t)\right) \\
& w_{s(i, j)}(t+1)=w_{s(i, j)}(t)+\eta(t) h(t) S-w_{s(i, j)}(t) .
\end{aligned}
$$

Step 5.5: Increment the iteration number $t$ and then update the value of $\eta(t)$ by equation (5) and $\sigma(\mathrm{t})$ by equation 6 :

$$
\begin{aligned}
& \eta(t)=\eta_{0} \exp \left(-\frac{t}{\lambda}\right) \\
& \sigma(t)=\sigma_{0} \exp \left(-\frac{t}{\lambda}\right)
\end{aligned}
$$

where $\lambda$ is a constant related to the maximum number of iterations and the size of the initial neighbourhood variance. It is given by:

$$
\lambda=\max -\text { iterations } / \log \left(\sigma_{0}\right) .
$$

Step 5.6: Repeat steps 5.1-5.5 until the stopping criteria is satisfied. The stopping criterion is the maximum number of epochs or when the convergence criterion given in equation (8) is met:

$$
e=\frac{1}{N} \sum_{i=1}^{N}\left\|w_{i, j}(t)-w_{i, j}(t+1)\right\|^{2} \leq e_{\min } .
$$

Step 6: Select the BMUs which are inside and located closer to the border of the search boundary for the colour to be segmented from the grid of nodes clustered after training. The weight vector of the hue specifies the new upper and lower limits within the search boundary while the weight vector of the saturation specifies the lower limit of the search area. The upper limit of the saturation is always given by the normalised value 255 .

Step 7: Segment the image on the basis of the selected BMU's in step 6. Use the if-then rule for segmentation. 
The training process divides the grid of nodes into most dominant colours depending on the size of the grid. For example if the grid size is 3, a maximum of 9 dominant colours present in the test image are obtained. These nine colours can then be used to assign the boundary values for the colour under consideration through its BMU's obtained by the proposed approach. The BMU's have their own associated weight vectors which can be used to segment the image. Therefore the larger the size of the grid the greater the number of representative colours which will be considered and thus better segmentation can be achieved.

\section{Experiments and Result Analysis}

This section deals with the experiments and results achieved by the adaptive colour segmentation algorithm. All the images of traffic signs used in these experiments were obtained from the set of images collected by Fleyeh [5]. The database contains images of different sizes. However, the size of all test images is either $640 \times 480$ pixels from the original or normalised to $640 \times 480$.

For the purpose of this paper, images were grouped into two sets:

- Set 1 consists of red-rimmed traffic sign images which were collected in different light and weather conditions.

- Set 2 consists of red-rimmed traffic sign images which were collected in different countries.

In all of the experiments described in this paper, 1 of the pixels in the image to be segmented were used for training and the quality of segmentation was judged by a human observer. Since all images are $640 \times 480$ pixels, the number of pixels in the image is 307,200 pixels, which means that 3072 pixels are used for training. These pixels are selected randomly and without repetition. Therefore, the convergence criterion is set to be either when all of the 3072 pixels are used for training or when the error becomes less than $e_{\min }$.

\subsection{Effect of Environmental and Physical Conditions}

The current approach has been tested on 267 images collected in different environmental and physical conditions. The successful segmentation rates of each category are listed in Table 1. A comparison with a static Shadow and Highlight Invariant algorithm developed earlier [4], which will be referred to as the Comparison Algorithm, is also shown in this table. By observing these results, the best improvement achieved by the proposed approach is for the faded signs which are segmented properly even when the sign is very faded and has only a small amount 


\begin{tabular}{|l|c|c|c|c|}
\hline \multirow{2}{*}{ Effect } & \multicolumn{2}{|c|}{ Adaptive Segmentation } & \multicolumn{2}{c|}{ Comparison Algorithm } \\
& $\begin{array}{c}\text { No. of } \\
\text { Signs }\end{array}$ & $\begin{array}{c}\text { Seg. Rate } \\
\text { \% }\end{array}$ & $\begin{array}{c}\text { No. of } \\
\text { Signs }\end{array}$ & $\begin{array}{c}\text { Seg. Rate } \\
\%\end{array}$ \\
\hline Bad Lighting & 50 & 90 & 48 & 87 \\
\hline Faded & 86 & 73 & 45 & 53 \\
\hline Blurred & 30 & 97 & 40 & 97 \\
\hline Fog & 13 & 75 & 27 & 74 \\
\hline Highlight & 36 & 97 & 40 & 97 \\
\hline Rainfall & 27 & 100 & 44 & 95 \\
\hline Snowfall & 25 & 92 & 44 & 89 \\
\hline
\end{tabular}

Table 1. Summary of the analysis and comparison.

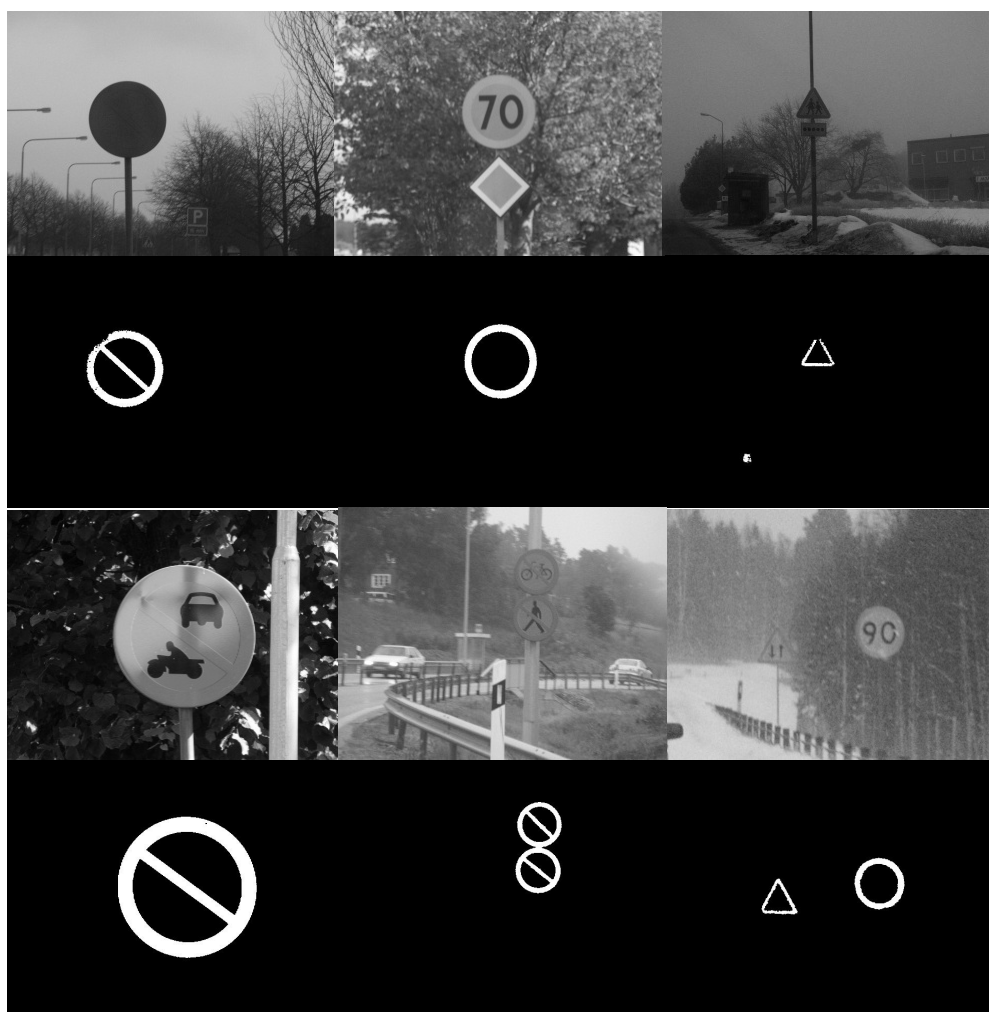

Figure 6. Segmentation results of traffic sign images. Top Left to Bottom Right: Bad lighting, faded signs, fog, highlight effect, rain fall, and snow fall. 
of red colour in it. For all other categories, the results achieved by the proposed approach are either better or the same. The main reason for good segmentation is the fixing of a proper search boundary from where the BMUs are picked for the segmentation and then the assignment of a dynamic boundary to reduce the segmented area in the final output. The algorithm failed to segment the traffic signs which were badly damaged in terms of colour and their red component was nearly lost due to fading. Other reasons of non segmentation included poor lighting or with multiple conditions such as faded signs in bad lighting. Figure 6 depicts the results of segmentation for this category of images.

\subsection{Segmentation in Different Countries}

Traffic sign images collected from nine countries were used to check the adaptability of the current approach for different countries. The total number of images used for testing the approach was 175 images. They were collected in seven European countries, the USA and Japan. The complete list of countries, the number of images and the segmentation rate are listed in Table 2.

The proposed algorithm gave very good results for the signs from different countries compared with the Comparison Algorithm. The variation of colour in these different countries was the potential difficulty but the results proved that the dynamic segmentation carried out by the BMUs gives very good performance in the segmentation. The original and segmented images show that the algorithm performs well even for signs observed from a far distance. The algorithm failed in those images which either were very dim or had their colour outside the maximum search space. Figure 7 depicts results of segmentation for this category of images.

\begin{tabular}{|l|c|c|c|}
\hline \multicolumn{1}{|c|}{ Country } & $\begin{array}{c}\text { No. of } \\
\text { Images }\end{array}$ & $\begin{array}{c}\text { Segmentation Rate } \\
\text { of Proposed } \\
\text { Algorithm \% }\end{array}$ & $\begin{array}{c}\text { Segmentation Rate } \\
\text { of Comparison } \\
\text { Algorithm \% }\end{array}$ \\
\hline Austria & 28 & 100 & 82.1 \\
\hline France & 13 & 100 & 61.5 \\
\hline Germany & 14 & 100 & 92.8 \\
\hline Japan & 12 & 100 & 75.0 \\
\hline The Netherlands & 15 & 100 & 93.3 \\
\hline Poland & 30 & 90 & 60.0 \\
\hline Spain & 25 & 92 & 68.0 \\
\hline The UK & 28 & 100 & 92.8 \\
\hline The USA & 10 & 80 & 70 \\
\hline
\end{tabular}

Table 2. Result of Colour Segmentation for Different Countries. 


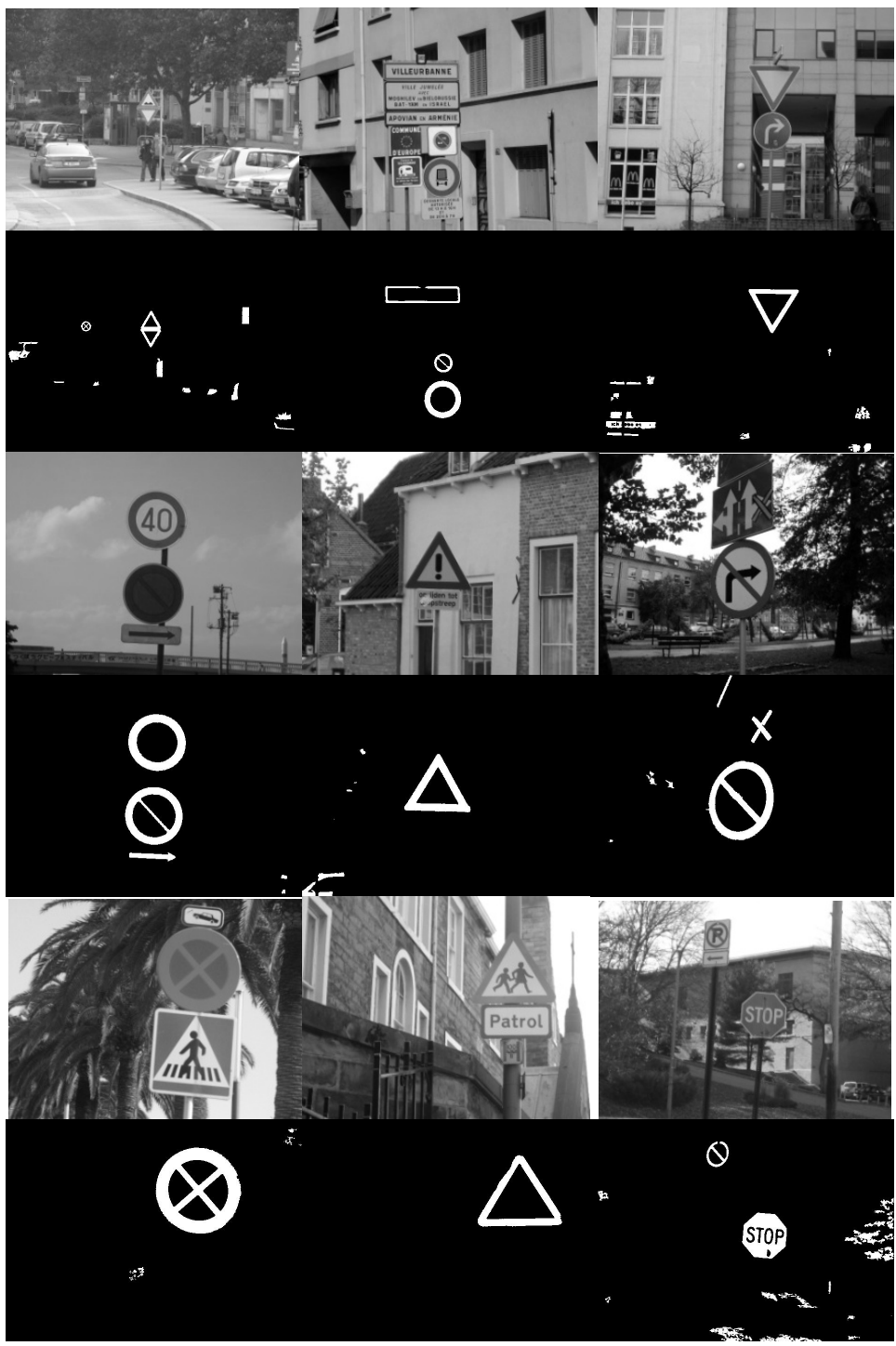

Figure 7. Traffic sign images and their segmented images. Top Left to Bottom Right: Austria, France, Germany, Japan, The Netherlands, Poland, Spain, The UK, and The USA. 


\subsection{Performance of the Proposed Approach}

The proposed approach was tested using a set of images collected from different distances. The images in Figure 8 were collected in $10 \mathrm{~m}$ steps from the traffic sign which gives different sizes of traffic signs. They contain noisy background which is similar to the traffic sign's colour. Segmentation was implemented using $8 \times 8$ and $16 \times 16$ grids. The execution time of the segmentation algorithm, which was implemented using Matlab and Dell Latitude E6400 with Intel Core Duo CPU $2.53 \mathrm{GHz}$ processor, was measured for each image. Figure 9 depicts the time required for segmentation in both cases. The plot shows a constant execution time regardless of the size of the traffic sign in the image or the amount of background noise. The result is compared with that of the Comparison Algorithm which shows great variation in the execution time for this algorithm. This variation is due to the fact that its execution time is related to how much colour is available in the image under consideration.

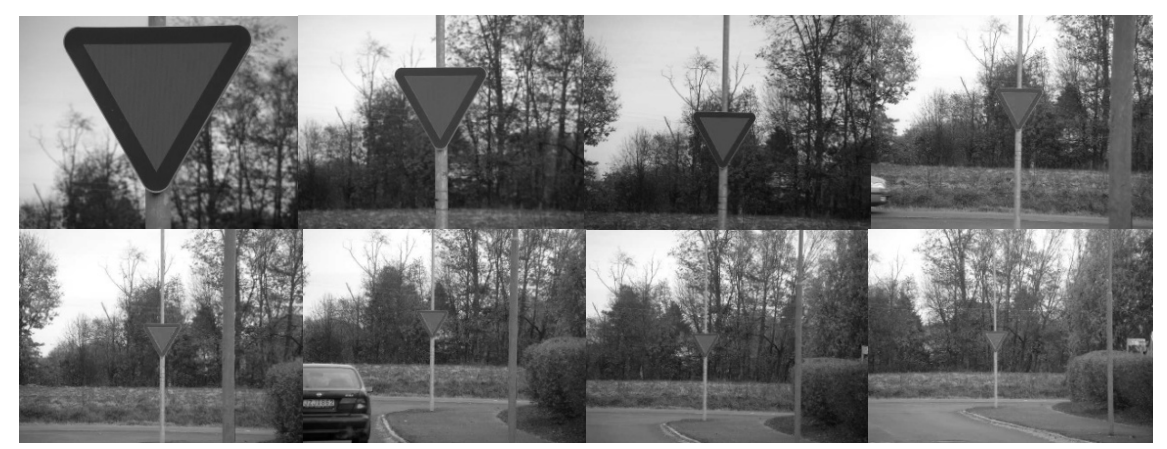

Figure 8. Images of the same sign taken in eight different distances.

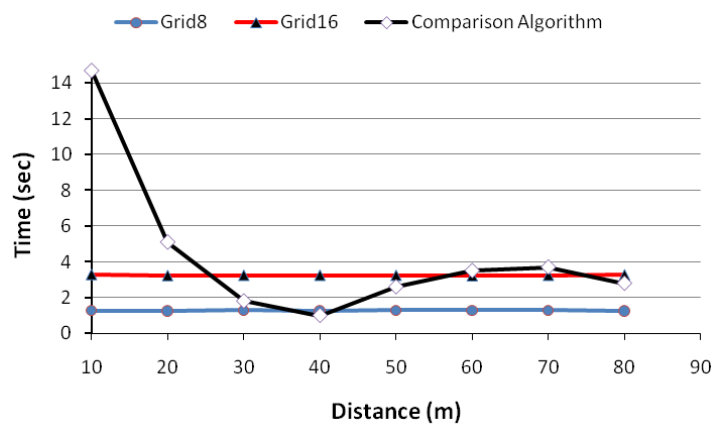

Figure 9. Execution time of same traffic sign versus different distances. 


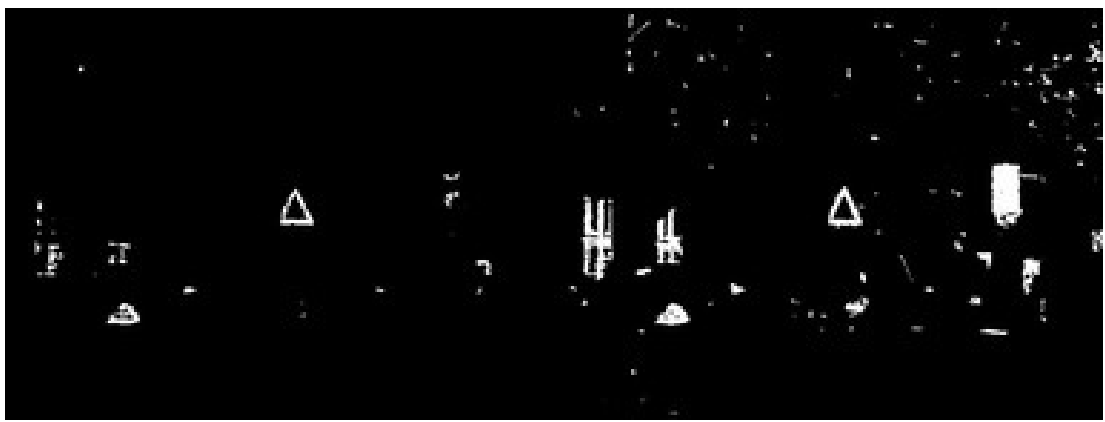

Figure 10. Comparison of segmentation by the proposed approach (left) with the comparison algorithm (right).

The average time for an $8 \times 8$ grid is 1.3 second compared with 3.2 seconds for a $16 \times 16$ grid and 4.4 seconds for the Comparison Algorithm. The standard deviation for the $8 \times 8$ grid is 0.015 compared with 0.025 for the $16 \times 16$ grid and 4.34 for the Comparison Algorithm. This shows the superiority of the proposed approach to achieve colour segmentation regardless of the size of the sign in the image or the distance from the camera. This algorithm produces less noise objects in the output image compared with the Comparison Algorithm which means better classification results at a later stage. Figure 10 depicts a comparison between the segmentation achieved by the proposed approach compared with the Comparison Algorithm.

\section{Conclusion and Future Work}

This paper proposed an intelligent algorithm for colour segmentation of traffic signs. Traditional colour segmentation algorithms often do not have fast, accurate and adaptive training steps. The proposed intelligent algorithm showed fast convergence to a satisfactory number of nodes which represent dominant colours of the segmented image and it is accurate as it gave fewer segmented regions compared with the traditional approach. Moreover, it is adaptive as it addressed and solved three major problems which are colour variations in outdoor images due to changes of lighting, colour changes of traffic signs due to aging, and colour changes because of different colour standards in different countries.

The proposed approach has produced better results compared to the traditional approach in all conditions especially for faded signs in which the proposed algorithm gave $73 \%$ in comparison with $53.3 \%$ of its counterpart. This is due to its 
ability to segment the test images dynamically and adaptively. The adaptive nature is also proved from the performance of the algorithm observed in the segmentation of images from different countries. The algorithm fails in conditions where the desired colours are faded so that the colour is not located in the chromatic area any longer.

A total of 442 images collected in different conditions and different countries were used for testing. The results showed that using a small percentage of the image, $1 \%$ of the pixels, is enough to train the proposed approach.

Comparisons of the proposed approach with one of the traditional techniques showed the superiority of the proposed approach in all conditions used for testing. The algorithm achieved better or at least equal success of segmentation in all images used for testing. More research is needed to be done for segmentation of objects subjected to conditions such as dim light with fog or objects subjected to hard environmental effects such as fog and snowfall. Clustering based on shapes instead of colours is another area for future work. This is interesting due to the fact that it will reduce the overhead of the system to differentiate between different conditions and work in all types of conditions even in very low illumination, by just searching for the shapes in a particular search space. This can reduce the problem being faced due to different conditions for Intelligent Transport System (ITS). This can bring dynamic improvements for the ITS.

\section{Bibliography}

[1] A. Araujo and D. Costa. Local adaptive receptive field self-organizing map for image color segmentation. Image and Vision Computing 27 (2009), 1229-1239.

[2] S. Buluswar and B. Draper. Non-parametric classification of pixels under varying outdoor illumination. ARPA Image Understanding Workshop, 1994.

[3] S. Buluswar and B. Draper. Color recognition in outdoor images. Inter. Conf. Computer vision, Bombay, India, 1998.

[4] H. Fleyeh. Shadow And Highlight Invariant Colour Segmentation Algorithm For Traffic Signs. 2006 IEEE Conf. on Cybernetics and Intelligent Systems, Bangkok, Thailand, 2006.

[5] H. Fleyeh. Traffic Signs Database, from http://users.du.se/ hfl/traffic _signs/ (2009, 1/9/2010).

[6] T. Gevers and A. Smeulders. Color-based object recognition. Pattern Recog. 32 (1999), 453-464.

[7] T. Kohonen. Self-Organizing Maps. Berlin, Heidelberg, New York, Springer-Verlag, 2001. 
[8] M. Prieto and A. Allen. Using self-organising maps in the detection and recognition of road signs. Image and Vision Computing 27 (2009), 673-683.

[9] A. Soetedjo and K. Yamada. A new approach on red color thresholding for traffic sign recognition system. Journal of Japan Society for Fuzzy Theory and Intelligent Informatics 19(5) (2007), 458-465.

[10] S. Vitabile, A. Gentile et al. A neural network based automatic road sign recognizer. The 2002 Inter. Joint Conf. on Neural Networks, Honolulu, HI, USA, 2002.

[11] Y. Wu, Q. Liu et al. An Adaptive Self-Organizing Color Segmentation Algorithm with Application to Robust Real-time Human Hand Localization. Asian Conf. on Computer Vision. Taiwan, 2000.

[12] N. Yeo, K. Lee et al. Color image segmentation using the self-organizing map and adaptive resonance theory. Image and Vision Computing 23(12) (2005), 1060-1079.

Received September 21, 2010.

\section{Author information}

Hasan Fleyeh, Department of Computer Engineering, School of Technology and Business Studies, Dalarna University, Borlänge, Sweden.

E-mail: hfl@du.se

Al-Hasanat R. M. Bin Mumtaz, Department of Computer Engineering,

School of Technology and Business Studies, Dalarna University, Borlänge, Sweden.

E-mail: hasanatrasul@gmail.com 